\title{
Birth Prevalence of Congenital Cytomegalovirus (CMV) Infection in a Cohort of Pregnant Women in Bangladesh
}

\author{
Jahan $\mathrm{M}^{1}$, Sultana $\mathrm{N}^{1}$, Asma $\mathrm{R}^{1}$, Tabassum $\mathrm{S}^{1}$, Islam $\mathrm{MN}^{1}$ \\ Department of Virology, Bangabandhu Sheikh Mujib Medical University, Dhaka, Bangladesh \\ e-mail: mjahan1970@yahoo.com
}

\begin{abstract}
Cytomegalovirus (CMV) is a frequent cause of congenital infection in humans in all regions of the world. In contrast to most congenital viral infections, congenital CMV infection and disease have been consistently demonstrated in populations with a high seroprevalence. Three hundred pregnant women were studied prospectively in their 1st, 2nd and 3rd trimester to determine the seroprevalence and seroconversion of CMV in pregnancy. After birth, babies were also tested for anti CMV IgM to determine the rate of birth prevalence. Anti CMV IgG and IgM tests were performed by chemiluminescence methods. All 300 (100\%) pregnant women were anti CMV IgG positive and $180(60 \%)$ were subsequently anti CMV IgM positive during different trimesters of pregnancy. Birth prevalence of CMV IgM antibody was $1.3 \%$ among babies of anti CMV IgM positive mothers whereas none in CMV IgM negative mothers (OR 1.01, 95\% CI .996-1.027). It may be concluded that CMV IgG seroprevalence is high among Bangladeshi pregnant women and the rate of CMV reactivation is also high during pregnancy. Despite protection by maternal immunity a certain percent of babies acquire congenital CMV infection.
\end{abstract}

Keywords: Birth prevalence of CMV, CMV IgM antibody, CMV IgG antibody

\section{Introduction}

Cytomegalovirus (CMV) is a common cause of congenital infection in humans in all regions of the world, with an incidence between $0.3 \%$ and $2.4 \%$ of all live births. ${ }^{1,2}$ Infection is more prevalent in underdeveloped countries and among lower socioeconomic groups in developed countries. ${ }^{1}$ Approximately 10 to $15 \%$ infants infected with congenital CMV exhibit clinically apparent or symptomatic form of the disease, characterized by petechiae, hepatomegaly, splenomegaly, jaundice, periventricular calcifications, microcephaly, hearing impairment and chorioretinitis. The remaining 85 to $90 \%$ infected infants are asymptomatic at birth, but $15 \%$ of them will develop delayed sequel like sensorineural hearing loss, mental retardation, and neurologic deficits. ${ }^{3-5}$ In contrast to most congenital viral infections, congenital CMV infection and disease can occur in children born to women with preexisting immunity to $\mathrm{CMV}^{6}$ The incidence of congenital infection depends on epidemiological characteristics of the population, in particular, the maternal CMV seroprevalence.
Presence of maternal antibody to CMV before conception provides substantial protection against intrauterine transmission of virus and severe foetal infections. Protection, however, is incomplete, and congenital CMV infection may follow recurrent maternal infection. ${ }^{6,7}$ Symptomatic congenital CMV infection after a recurrent maternal infection occurs more frequently than previously documented. ${ }^{6-8}$ This study was designed to determine the seroprevalence and seroconversion of CMV in pregnancy as well as the birth prevalence of congenital CMV infection among neonates in a cohort of pregnant women of Bangladesh.

\section{Material and Methods}

This cross sectional, prospective study was conducted during July 2013 to June 2014. A total of 700 pregnant women were interviewed attending the outpatient Department of Gynaecology and Obstetrics, Bangabandhu Sheikh Mujib Medical University (BSMMU), Dhaka for their anti-natal checkup. From them, 600 pregnant women were selected according to selection criteria. Among 600 pregnant women 
300 were randomly recruited by table of random numbers (every $2^{\text {nd }}$ patients were included in the study). After taking informed written consent approximately $5 \mathrm{ml}$ venous blood was collected with all aseptic precautions to assess the anti CMV IgG and IgM status in their pregnancy. Serum was separated and stored at $-20^{\circ} \mathrm{c}$ until tested. All of them were followed up in every trimester till delivery to determine their CMV antibody status. Babies were also tested for anti CMV IgM to determine the rate of birth prevalence of congenital CMV infection. Anti CMV IgG and IgM tests were performed in the Department of Virology, Bangabandhu Sheikh Mujib Medical University, Dhaka using chemiluminescence method (LKCV1, LKCM1, Siemens, UK) according to the manufacturer instructions. This method was performed in Immulite Automated Immunoassay System (DPC Immunlite, Germany 49-6032-994-00. Certificate No: A3493. ISO 13485:1996).

CMV IgG and IgM assay was based on solid phase chemiluminescent enzyme immunoassay. The DPC Immulite is an automated random access immulite analyzer. CMV IgG and IgM assay utilized the specific antigen coated polystyrine bead as the solid phase. The bead was dispensed in a reaction tube (test unit) which served as the vessel for the incubation, reaction, wash, and signal development processes. Diluted sample was dispensed in the sample cup and placed into bar coded sample cup holder. Sample cup holders were placed on the load platform of Immulite serially and bar coated test units were placed after each of the sample cup holder. Then the sample was dispensed into the test unit (automatically) and allowed 30 minutes incubation. Unbound material was then removed by spinning the test unit at high speed. The fluid contents were transferred to a co-axial space of the test unit. The alkaline Phosphatase-labeled monoclonal murine anti human $\operatorname{IgG}$ was then added and another 30 minutes incubation was followed. Once the test unit was washed, a luminogenic substrate, a phosphate ester of adamentyldioxitane was added. In the luminogenic reaction the substrate was dephosphorylated into an unstable anion intermediate by the alkaline phosphatase conjugate captured on the bead. The unstable intermediate emits a photon upon decomposition.
The photon counts were measured with a photomultipelier tube (PMT) as counts per second (CPS) were converted to analyte concentrate using stored master curves, printed report from each sample was generated by the system computer.

SPSS (version 21) software was employed to analyse the data. Categorical variables were analysed using Chi square test, while logistic regression test was used to calculate adjusted ORs of CMV seroprevalence in successive bivariate models. Statistical level of significance was considered at $p<0.05$.

\section{Results}

The demographic characteristics of the study population are shown in table I. The mean age was 25.01 years and their age range was18-38 years. Data showed that there was no significant association between the age of study subjects and the prevalence of anti CMV-IgM positivity $(p=0.128)$. Prevalence of anti CMV-IgM positivity was more among 21-30 years age group which was not statistically significant. Prevalence of anti CMV-IgM positivity was significantly higher in pregnant women from urban area $(p=0.05)$. Majority of the pregnant women of this study were Muslims (94.3\%), housewives $(88.3 \%)$, residents of urban areas $(81 \%)$ and $68 \%$ of them had more than 5 years of schooling (table I).

Table I. Demographic Characteristics of pregnant women $(\mathrm{n}=300)$

\begin{tabular}{lll}
\hline Characteristics & Categories & Frequency $(\%)$ \\
\hline Age & $10-20 y r s$ & $71(23.7)$ \\
& $21-30 y r s$ & $195(65.0)$ \\
& $31-40 y r s$ & $34(11.3)$ \\
Demographic & Rural area & $57(19)$ \\
area & Urban area & $243(81)$ \\
Religions & Islam & $283(94.3)$ \\
& Hindu & $17(5.6)$ \\
Women's & No formal education & $39(13)$ \\
Education & $1-5$ years of schooling & $57(19)$ \\
& 5-10 years of & $103(34.3)$ \\
& schooling & $101(33.7)$ \\
& More than 10 years of & \\
Women's & education & $265(88.3)$ \\
Occupation & Service Provider & $35(11.7)$ \\
\hline
\end{tabular}

Amongst the pregnant women 47 (15.67\%) were of parity $\geq 3,182(60.66 \%)$ of parity $1-2$ while $71(23.67 \%)$ were primigravidae. There was no 
association between parity/gravida and birth prevalence of CMV $(p=0.95)$.

Data showed that there was no association between socioeconomic status and CMV infection (anti CMV IgM positivity) among pregnant women $(p=0.287)$. Prevalence of CMV infection (anti CMV IgM positivity) was only $5.7 \%$ in upper class group (figure I).

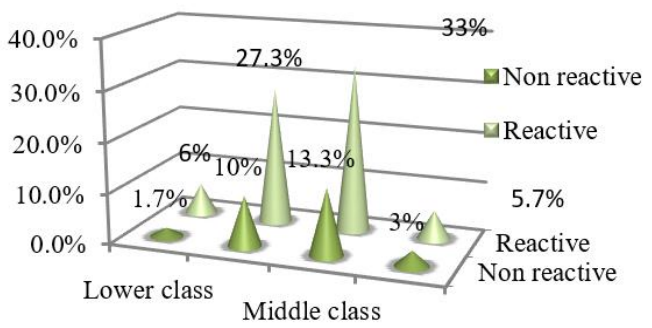

Figure I: Anti CMV IgM status in different socioeconomic status of pregnant women $(n=300)$

Among the total 300 pregnant women,76.3\% women were of average nutrition and $17.3 \%$ were of under nutrition (figure II) and there was no significant association between nutritional status and CMV infection among pregnant women $(p=0.07)$. No obese (BMI of 30 or higher) pregnant women was to be found among the study population.

\section{Nutritional status}

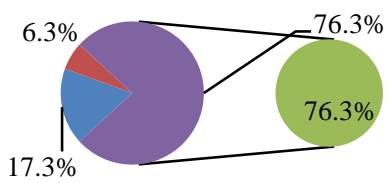

- Under nutrition

n Over nutrition

average nutrition

Figure II: Nutritional status of pregnant women $(n=300)$

All 300 pregnant women were anti CMV $\operatorname{IgG}$ positive on their first visit. Among them, 180 (60\%) were subsequently anti CMV IgM positive during different periods of their pregnancy. Among these 180 anti CMV IgM positive women $131(43.7 \%)$ were positive in the first trimester, $35(11.7 \%)$ during their second trimester and $14(4.6 \%)$ were positive during their third trimester. Birth prevalence of CMV IgM antibody was $1.3 \%$ among babies of anti CMV IgM positive mothers whereas none in CMV IgM negative mothers (OR 1.01, 95\% CI .996-1.027). A significant $(p<0.001)$ association of anti CMV-IgM positivity was demonstrated among anti CMV IgM positive mothers and their babies (table II).

\begin{tabular}{|c|c|c|c|c|c|c|}
\hline \multirow{2}{*}{$\begin{array}{c}\text { Status } \\
\text { of Anti } \\
\text { CMV-IgM } \\
\text { among } \\
\text { mothers } \\
\mathrm{N}=300\end{array}$} & \multicolumn{2}{|c|}{$\begin{array}{l}\text { Anti CMV-IgM } \\
\text { status among } \\
\text { babies }\end{array}$} & \multirow[t]{2}{*}{ Total } & \multirow[t]{2}{*}{$P$ value } & \multirow[t]{2}{*}{$\mathrm{OR}^{*}$} & \multirow[t]{2}{*}{$\begin{array}{c}95 \% \\
\mathrm{CI}\end{array}$} \\
\hline & $\begin{array}{l}\text { Non- } \\
\text { reactive }\end{array}$ & Reactive & & & & \\
\hline $\begin{array}{c}\text { Non- } \\
\text { reactive }\end{array}$ & $\begin{array}{c}120 \\
(40 \%)\end{array}$ & $0(0 \%)$ & 120 & & & \\
\hline Reactive & 176 & $4(1.3 \%)$ & 180 & & & \\
\hline & $(58.7 \%)$ & & & 0.001 & 1.02 & $(1.000$ \\
\hline Total & 296 & $4(1.3 \%)$ & $300(1$ & & & - \\
\hline & $(98.7 \%)$ & & $00 \%)$ & & & 1.046) \\
\hline
\end{tabular}

\section{Discussion}

Cytomegalovirus (CMV) infection is a common viral infection in pregnancy. ${ }^{9}$ Percentage of seropositivity varies among pregnant women from different countries and socioeconomic groups. A rough correlation exists between the prevalence of antibody and the socioeconomic conditions of the population. The prevalence of antibody is significantly higher in developing countries of Africa and Southeast Asia, whereas it is low in Europe, Australia, and parts of North America. ${ }^{9}$ Various studies have demonstrated that the prevalence of antibody is significantly higher (85-100\%) in India, 87.8\% in Japan, 82\% in Alabama, $100 \%$ in Entebbe and Fiji, whereas $57 \%$ in Washington USA and $44 \%$ in. Oxford UK. ${ }^{10-13}$ Serological surveys from different parts of India have shown $80-90 \%$ prevalence of CMV IgG antibodies in women of childbearing age. ${ }^{14}$ Satilmis et al showed the rate of seroprevalence was $98.5 \%$ among pregnant woman from Turkey. ${ }^{15}$ This study demonstrated that $100 \%$ pregnant women were seropositive in their first antenatal visit, reflecting a high seroprevalance in Bangladesh. High seroprevalence (97\%) was also observed in another study from Bangladesh. ${ }^{16}$ Redwan et al showed $83.6 \%$ prevalence in Bagladeshi population. ${ }^{17}$ Ashrafunnesa et al observed slightly lower but still a high prevalence $(68.6 \%)$ in antenatal population in Bangladesh. Socioeconomic status, level of education might be responsible for the high prevalence of 
CMV. ${ }^{18}$ CMV prevalence among pregnant women might also be affected by having children. ${ }^{19,20}$ In this study, $91.3 \%$ pregnant women belong to middle class and below middle class groups, $66.3 \%$ had $\leq 10$ years of schooling and $76.33 \%$ had children.

Pregnancy is associated with a functional immunosuppression and, thus, may increase the risk of acquiring infections. ${ }^{21}$ Due to latency and periodic reactivation of $\mathrm{CMV}$, transmission in utero may follow either primary or reactivation of latent infection. This study demonstrated that among all the CMV IgG seropositive women $180(60 \%)$ became CMV IgM positive during some point of their pregnancy. As CMV seroprevalance is high in Bangladesh and mothers were already CMV IgG positive, these $180(60 \%)$ cases may be considered as reactivation of CMV during pregnancy. The prevalence of congenital infection was $1.3 \%$ in this study. In India congenital CMV infection was observed in $2.4 \% .{ }^{8}$ In a study from Sapporo, Japan, the rate of congenital infection was reported to be $0.31 \%$. Of them, $13.5 \%$ were symptomatic at birth, 5 to $17 \%$ of them were likely to develop progressive sensorineural hearing hearing loss within their first 4 years of life. ${ }^{22}$

Congenital infection due to primary maternal infection is usually associated with more severe sequel. ${ }^{6}$ Cytomegalovirus infection frequently occurs despite substantial humeral immunity in mothers, which is most often due to reactivation of latent virus. ${ }^{23,24}$ In fact, in some populations intrauterine cytomegalovirus infections are predominantly associated with reactivation of maternal infections. Stagno et al reported that the prevalence of congenital infection was $3.4 \%$ in an immune population. ${ }^{12}$ Despite the apparent lack of protection, maternal immunity may still exert a beneficial effect by reducing the virulence of infection in the fetus and developing infant. Recently it was demonstrated that reactivation or recurrent infection can cause severe fetal sequel despite maternal preexisting immunity. It has been suggested that the possibility of recurrences and unfavourable outcome may be related to reinfection by a new viral strain. ${ }^{7} \mathrm{~A}$ study from India reported that all $9(2.1 \%)$ CMV positive infants were born to mothers who were CMV seropositive at the first antenatal visit. ${ }^{8}$ Among them one child had hepatosplenomegaly at birth while another child had mild unilateral hearing loss at 4 months of age.Another study from United Kingdom also showed symptomatic CMV infection at birth $(18 \%)$ in infants of the primary-infection group. After a mean follow-up of 4.7 years, one or more sequelae were seen in $25 \%$ of the primaryinfection group and in $8 \%$ of the recurrentinfection group. Sensorineural hearing loss was found in 15 percent of those in the primaryinfection group and in only $5 \%$ of those in the recurrent-infection group. Bilateral hearing loss was identified only among children in the primary-infection group $(8 \%){ }^{6}$

\section{Conclusion}

Based on this study, it may be concluded that CMV IgG seroprevalence is high among Bangladeshi women, and the rate of CMV reactivation is also high during pregnancy. Despite the protection of maternal immunity, a certain percentage of babies acquire congenital CMV infection. In this context, it may be recommended that a screening program for CMV IgG and IgM antibodies should be conducted at the first antenatal visit. Considering the high risk of mother-fetus transmission and fetal damage, prenatal diagnosis should be recommended to women with primary CMV infection. To determine the manifestations and long term sequel of congenital CMV infection, conduction of longitudinal studies are thus suggested.

Conflict of interest: There is no conflict of interest about this article.

Acknowledgements: The study was supported by Bangabandhu Sheikh Mujib Medical University (BSMMU) under Health Nutrition \& Population Sector Development Programme (HNPSDP) Fund, Bangladesh.

\section{References}

1. Hanshaw J B. Congenital cytomegalovirus infection: A fifteen year perspective. J Infect Dis. 1971; 123:555-61.

2. Stagno S, Pass RF, Dworsky ME, Henderson RE, Moore EG, Walton PD et al. Congenital cytomegalovirus infection: The relative importance of primary and recurrent maternal infection. N Eng J Med. 1982; 306: 945-49. 
3. Alford CA, Stagno S, Pass RF, and Britt WJ. Congenital and perinatal cytomegalovirus infections. Rev Infect Dis. 1990; 12: s745-s53.

4. Numazaki K, Fujikawa T. Chronological changes of incidence and prognosis of children with asymptomatic congenital cytomegalovirus infection in Sapporo, Japan. BMC Infect Dis. 2004;4: 1-5.

5. Berenberg W, Nankervis G. Long term follow -up of cytomegalic inclusion disease of infancy. Pediatrics. 1970; 46:403-10.

6. Fowler KB, Stagno S, Pass RF, Britt WJ, Boll TJ, Alford CA. The outcome of congenital cytomegalovirus infection in relation to maternal antibody status. N Eng J Med. 1992;326:663-67

7. Boppana SB, Fowler KB, Britt WJ, Stagno S, Pass RF. Symptomatic congenital cytomegalovirus infection in infants born to mothers with preexisting immunity to cytomegalovirus. Pediatrics. 1999;104:55-60.

8. Dar L, PatiSK, Patro AR, Deorari AK, Rai S, Kant $S$ et al. Congenital cytomegalovirus infection in a highly seropositive semi-urban population in India. Pediatr Infect Dis J. 2008; 27:841-43

9. Kenneson A, Cannon MJ.Review and metaanalysis of the epidemiology of congenital cytomegalovirus (CMV) infection. Rev Med Virol. 2007;17:253-76

10. Pal SR, Chitkara NL, and Krech U. Seroepidemiology of cytomegalovirus infection in and around Chandigarh (Northern India). Ind J Med Res. 1972; 60: 973-78.

11. Sheevani, Jindal N, Aggarwal A.. A pilot seroepidemiological study of cytomegalovirus infection in women of child bearing age. Ind J Med Microbiol. 2005; 23: 34-36.

12. Stagno S, Reynolds DW, Huang E-S, Thames S D, Smith R J and Alford C A. Congenital cytomegalovirus infection: Occurrence in an immune population. N Eng J Med. 1977; 296: 1254-58

13. Krech U, Tobin J.. A collaborative study of cytomegalovirus antibodies in mothers and young children in 19 countries. Bulletin of the World Health Organization. 1981; 59: 605-10.

14. Gandhoke I, Aggarwal R, Lal S, Khare S. Congenital CMV infection in symptomatic infants in Delhi and surrounding areas. Indian $\mathrm{J}$ Pediatr. 2006; 73:1095-97.

15. Satilmiş A, Güra A, Ongun H, Mendilcioğlu I, Colak D, Oygür N. CMV seroconversion in pregnants and the incidence of congenital CMV infection.Turk J Pediatr. 2007;49:30-36.

16. Jahan M, TabassumS, Aziz A, Ahmed M, Islam MN.Transfusion associated CMV infection: Transfusion strategies for high risk patients. Bangladesh J Med Microbiol. 2010; 04: 24-27

17. N. A. Redwan, M. M. M. Ahmed and M. S. H. AL Awfi. Prevalence study of cytomegalovirus (CMV) infection among foreign manpower in Jeddah Saudi Arabia. African Journal of Microbiology Research. 2011; 5: 2539-49.

18. Ashrafun Nessa, Shahla S Khatun, Mohammad Nazrul MN Islam, M H MH Rashid. Seroprevalence of cytomegalovirus antibody in antenatal population in Bangladesh. Bangladesh Med Res Counc Bull. 2009; 35: 110-2.

19. Sheevani, Jindal N, Aggarwal A. A pilot seroepidemiological study of cytomegalovirus infection in women of child bearing age. Indian $\mathbf{J}$ Med Microbiol. 2005;23 34-36.

20. W. Wujcicka, Z. Gaj, J. Wilczyński, W. Sobala, E. Śpiewak, and D.Nowakowska.Impact of socioeconomic risk factors on the seroprevalence of cytomegalovirus infections in a cohort of pregnant Polish women between 2010 and 2011.Eur J ClinMicrobiol Infect Dis. 2014; 33: 1951-1958.

21. Tanaka A, Yamada H, Minami M et al Suppression of cell mediated immunity to cytomegalovirus and tuberculin in pregnancy employing the leukocyte migration inhibition test. Microbiol Immunol. 1983;27:937-43.

22. Numazaki K, Fujikawa T. Chronological changes of incidence and prognosis of children with asymptomatic congenital cytomegalovirus infection in Sapporo, Japan. BMC Infect Dis 2004; 4: 1-5.

23. Ahlfors K, Ivarsson S-A, Harris S, Svanberg L, Holmqvist R, Lernmark B, Theander G. Congenital cytomegalovirus infection and disease in Sweden and the relative importance of primary and secondary maternal infection. Scand J Infect Dis. 1984; 16: 129-37

24. Yeager AS, Martin HP, Stewart JA. Congenital cytomegalovirus infection outcome for the subsequent sibling. Clin Pediatrics. 1977; 16: 455-58. 